Scientific Paper

\title{
A simple multi-feature based stereoscopic medical image retrieval system
}

\author{
K.A. ShaheER AbubackeR ${ }^{1}$, J. Sutha ${ }^{2}$, K.A. ShahUl HAMEed ${ }^{3}$ \\ ${ }^{I}$ Anna University, Chennai, Tamilnadu, India \\ ${ }^{2}$ Department of CSE, AAA College of Engineering \& Technology, Sivakasi, Tamilnadu, India \\ ${ }^{3}$ Department of ECE, Sethu Institute of Technology, Pulloor, Kariapatti, Tamilnadu, India \\ ${ }^{a}$ E-mail address: contactabu@yahoo.com
}

(received 11 November 2018; revised 26 January 2019; accepted 14 February 2019)

\begin{abstract}
This paper describes a method of retrieving stereoscopic medical images from the database that consists of feature extraction, similarity measure, and re-ranking of retrieved images. This method retrieves similar images of the query image from the database and re-ranks them according to the disparity map. The performance is evaluated using the metrics namely average retrieval precision (APR) and average retrieval rate (ARR). According to the performance outcomes, the multi-feature based image retrieval using Mahalanobis distance measure has produced better result compared to other distance measures namely Euclidean, Minkowski, the sum of absolute difference (SAD) and the sum of squared absolute difference (SSAD). Therefore, the stereo image retrieval systems presented has high potential in biomedical image storage and retrieval systems.
\end{abstract}

Key words: stereoscopic image retrieval; feature extraction; similarity measure; disparity map; average retrieval precision; average retrieval rate.

\section{Introduction}

Recently, stereoscopic imaging systems have become popular in the field of biomedical sciences after the effective usages in breast cancer screening and diagnosis, and also guided surgeries [1,2]. In the therapeutic field, a large volume of stereo images needs to be stored as well as retrieved to give the required information at the right time in order to improve the quality and effectiveness of the clinical process. The contentbased image retrieval (CBIR) has been widely used in many applications to retrieve image information from the database that uses features such as keyword or tag and domain-specific features [3]. Several works related to medical CBIR have been shown in the literature [4,5]. However, most of the works aimed at a particular imaging modality and also task-specific [6].

Stereoscopic image retrieval (SIR) is considered as one of the most challenging works since they involve the nature of imaging device, modality, and complexity, and comparatively few works have been reported. For example, Ayoub Karine et al. have developed a new SIR system based on complex wavelet coefficient and evaluated the performance in terms of time, cost and the retrieval rates [7]. Yang Cao et al. have proposed an automatic tag saliency-ranking algorithm for stereo images [8]. In this paper, a novel method has been proposed to segment the two images into meaningful regions and then, the multi-instance learning algorithm is used to annotate tags on region-level. These tags are re-ranked according to 3-D saliency values. A. Chaker et al. proposed the two-retrieval method based on visual contents and the disparity information of the stereo images [9]. In this work, univariate and bivariate models are used separately to retrieve the images. The datasets used in most of the works are taken from natural scenes, objects and SPOT5 scenes. To the best of our knowledge, there is no work reported in medical SIR. In this paper, we have presented the SIR system for medical images. This paper is organized as follows: section II describes the methods used in the retrieval method; section III presents the performance outcomes and the conclusions are given in section IV.

\section{Methodology}

The system for medical stereoscopic image retrieval framework is shown in Figure 1. The dataset of medical stereo images has been created by Carestream workstation that consist of volume rendered MRI and CT anatomical images with different resolutions. The features such as color, texture and disparity map of all the images are extracted to create a feature database. In order to retrieve the image, the same features are obtained from the query image and compared with the feature vectors in the database. 


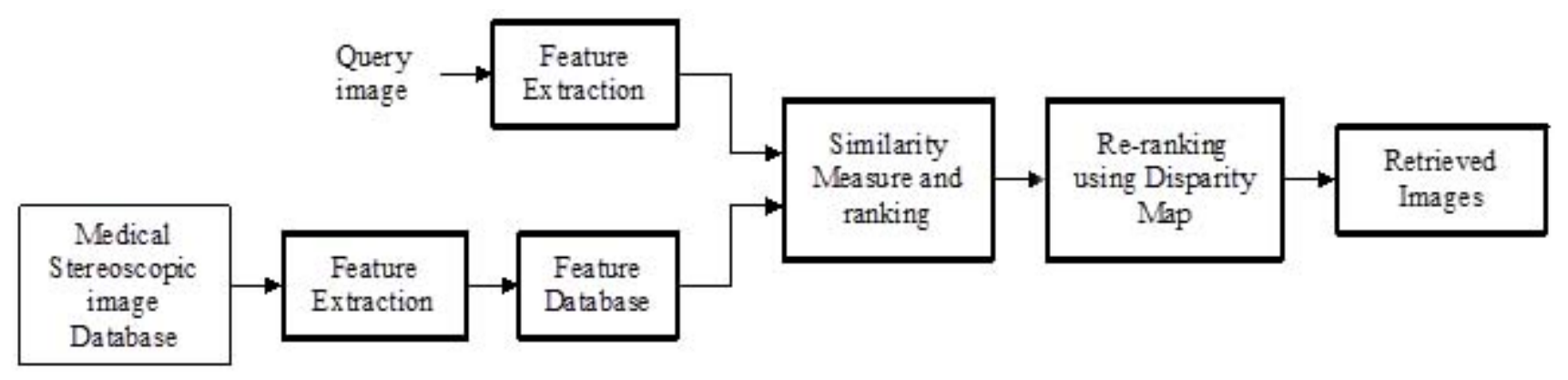

Figure 1. Block diagram of medical stereoscopic image retrieval.

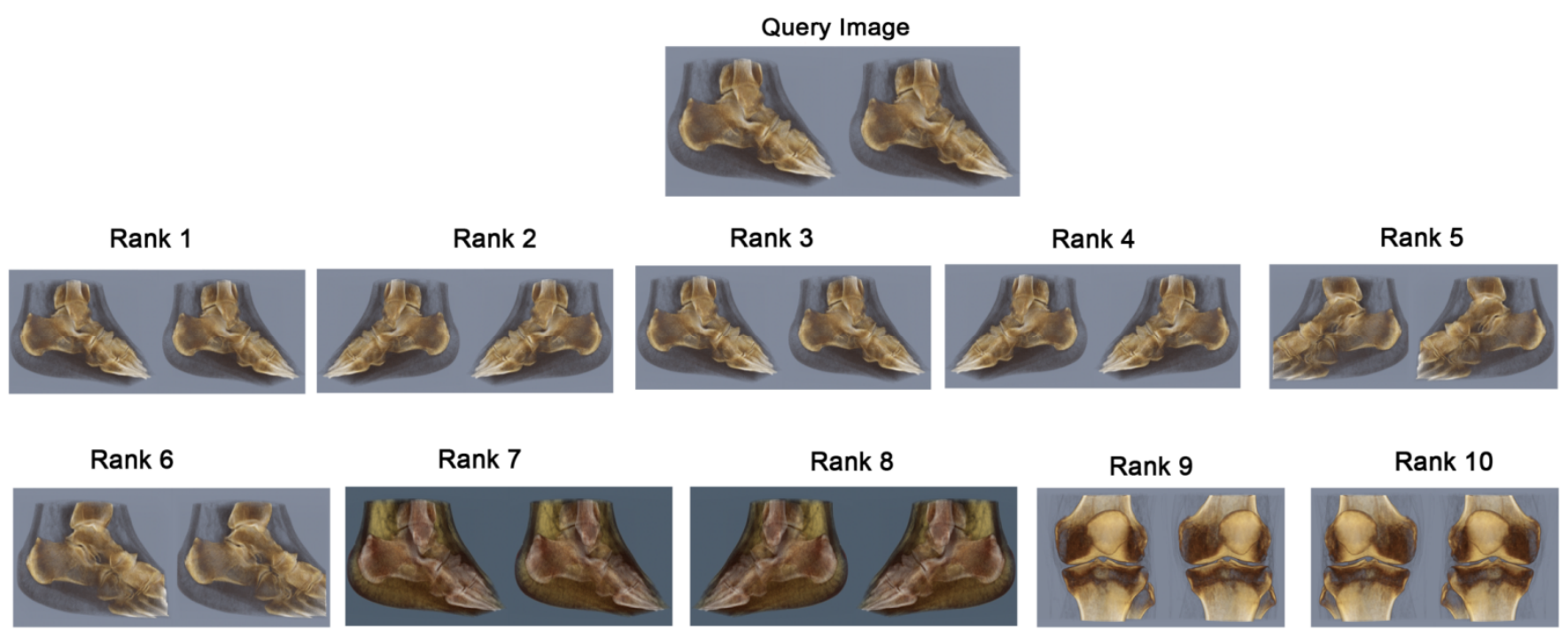

Figure 2. Retrieval results of proposed method on volume rendered stereoscopic database.

The similarity between the query image and database is determined and ranked according to highest similarity index value. Finally, the disparity map is used to re-rank the similar images for refining the result in order to increase the efficiency of the system.

\section{Feature extraction}

In general, the performance of an image retrieval system highly relied on the selection of feature extraction techniques and similarity measurement schemes. In this work, the features such as HSV color histogram [10], auto color correlogram [11], Gabor, wavelet [12] and Weber local descriptor [13] are extracted separately from left and right images. The disparity map [14] has been generated by determining differences between left and right images and then smoothed by applying the averaging filter.

\section{Similarity Index}

The similarity between the features query image and the database has been determined by comparing the feature vector of a query image and the database images.
Let $x_{q}=\left(x_{q 1}, x_{q 2}, \ldots . x_{q K}\right)$ is the feature vector of a query image and $x_{j}=\left(x_{j 1}, x_{j 2}, \ldots x_{j K}\right)$ is the feature vector of $j^{\text {th }}$ image in the database. The similarity between $x_{q}$ and $x_{j}$ is obtained as follows:

$S_{q j}=g\left(f_{1}\left(x_{q 1}, x_{j 1}\right), f_{2}\left(x_{q 2}, x_{j 2}\right) \ldots . . f_{2}\left(x_{q K}, x_{j K}\right)\right.$

Eq. 1

Where $f($.) gives the similarity between the individual feature vector and $g($.$) gives the weighted sum of the similarity vectors.$

The similarity index has been determined using the metrics namely Euclidean, Minkowski, Mahalanobis, SAD, and SSAD [15]. Based on the similarity value, the images in the database have been ranked. Further, the images ranked are refined using the disparity map of the query image and the ranked images as follows: Let $D_{q}$ and $D_{j}$ are the histograms of the estimated disparity map of the query image and $j^{\text {th }}$ image in the database. The consistency between $D_{q}$ and $D_{j}$, and $S_{q j}$ are then used to re-rank the retrieved images as follows:

$S_{q j}^{\prime}=\pi\left(D_{q}, D_{j}\right) S_{q j}$

Eq. 2

Here, $\pi($.$) is the distance between two functions.$ 


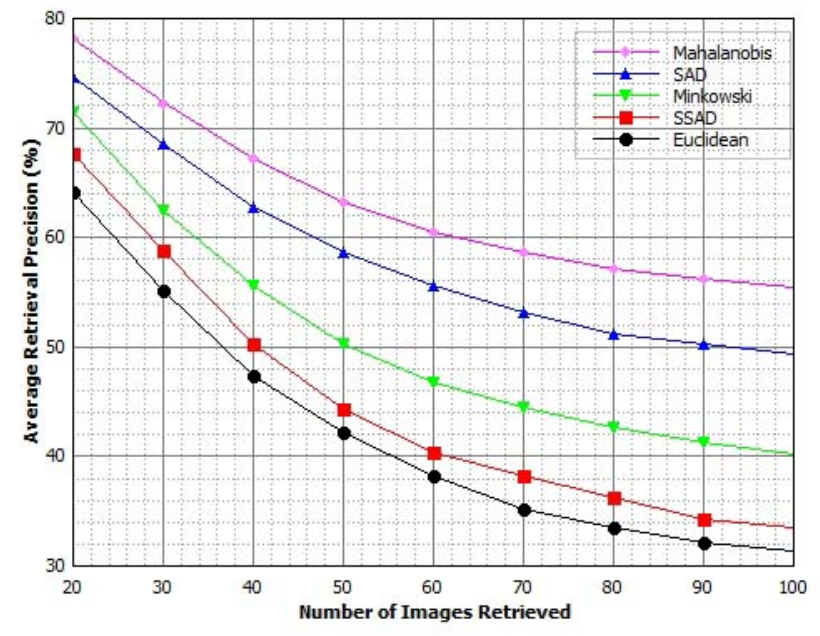

(a)

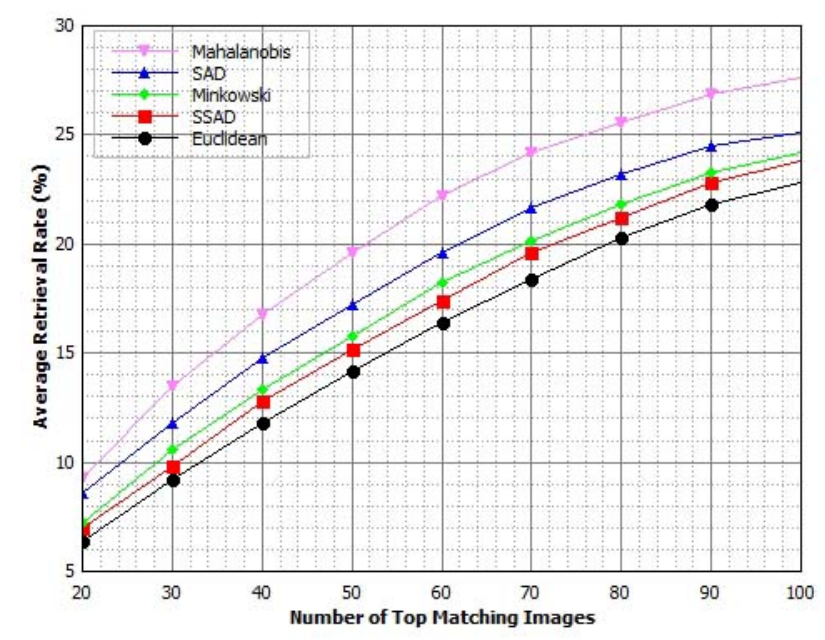

(b)

Figure 3. Comparison of retrieval results of Similarity Measure Methods in terms of Average Retrieval Rate and Average Retrieval Precision.

\section{Results}

The performance of the SIR method has been evaluated using the metrics namely precision $(\mathrm{P})$, average retrieval precision (APR), recall (R), and average retrieval rate (ARR) [16-18]. In this paper, a total of 850 stereogram images with different size and resolution are used in the evaluation process. The size of the image varies from $620 \mathrm{kB}$ to $9.5 \mathrm{MB}$ and resolution varies from $1430 \times 678$ to $3083 \times 1121$. These images are separated as per the organs namely brain, chest, arm, leg, stomach and nerves. The features are extracted from these images and stored in the feature database. During the image retrieval process, the features of the query image are obtained and compared with the database feature in order to determine the similarity index. The result is ranked according to the maximum similarity value. Further, the outcome is re-ranked using the disparity map. For example, a volume rendered CT scan image of Intraosseous lipoma in the calcaneus is used as a query image and the similar retrieved images are shown in Figure 2 in which the first ten images are displayed. The retrieved images are similar to the query image.

The performance metrics are measured for different query images and the corresponding retrieved images. Figure 3a shows the plot between the number of images retrieved and
ARP. According to the performance outcomes, Mahalanobis distance measure has produced ARP value of $78.2 \%$ when the number of the retrieved image is 20 . At the same time, the ARP value is slowly decreased when the size of the retrieved image set is increased. A similar scenario is noticed for other distance measures. However, the rate of reduction in ARP value is comparatively high when compared with distance measures namely Minkowski, SSAD, and Euclidean. Figure 3b shows the plot between the number of top matching images and ARR. Here, the Mahalanobis distance measure shows comparatively the best performance among other similarity measures.

\section{Conclusion}

In this paper, we have presented a stereoscopic image retrieval system for the medical application. This system consists of feature extraction, similarity measure, and re-ranking of retrieved images. The performance of the system has been evaluated by using the metrics namely APR and ARR. Based on the performance outcomes, the Mahalanobis distance measure has produced better results when compared to other distance measures. Therefore, the SIR systems described in this paper has a high value in the field of medical image retrieval.

\section{References}

[1] Getty DJ, D’Orsi CJ, Pickett RM. Stereoscopic digital mammography: Improved accuracy of lesion detection in breast cancer screening. In: Krupinski EA (ed) Digital Mammography. IWDM 2008. Lecture Notes in Computer Science, vol 5116. Springer, Berlin, Heidelberg. pp 74-79.

[2] Daul C, Graebling P, Tiedeu A, Wolf D. 3-D reconstruction of microcalcification clusters using stereo imaging: algorithm and mammographic unit calibration, IEEE Trans Biomed Eng. 2005;52(12):2058-2073. 
[3] Niblack CW, Barber R, Equitz W, et al. The QBIC Project: Querying Images by Content, Using Color, Texture, and Shape. SPIE Conf on Storage and Retrieval for Image and Video Databases. 1993;1908.173-187.

[4] Welter P, Riesmeier J, Fischer B, et al. Bridging the integration gap between imaging and information systems: a uniform data concept for content-based image retrieval in computer aided diagnosis. J American Med Informatics Association. 2011;18(4):506510 .

[5] Lehmann TM, Wein B, Dahmen J, et al. Content-based Image Retrieval in Medical Applications: A Novel Multi-step Approach. Proces of SPIE - The Int Society for Optical Engineering. 2000;3972:312-320.

[6] Smeulders AW, Worring M, Santini S, et al. Content based image retrieval at the end of the early years. IEEE Trans on Pattern Analysis \& Machine Intelligence. 2000;12:1349-1380.

[7] Karine A, El Maliani AD, El Hassouni M. A novel statistical model for content-based stereo image retrieval in the complex wavelet domain. J of Visual Comm and Image Representation. 2018;50:27-39.

[8] Cao Y, Kang K, Zhang S, et al. Automatic tag saliency ranking for stereo images. Neurocomputing. 2016;172:9-18.

[9] Chaker A, Kaaniche M, Benazza-Benyahia A. Disparity based stereo image retrieval through univariate and bivariate models. Signal Process. Image Comm. 2015;31:174-184.

[10] Gonzalez RC, Woods RE. Digital image processing, Prentice-Hall, Inc. Upper Saddle River, NJ, USA 2002.

[11] Huang J, Kumar SR, Mitra M, et al. Image indexing using color correlograms. Computer Vision and Pattern Recognition, 1997. Procs of 1997 IEEE Computer Society Conf on, IEEE. 1997;762-768.

[12] Shalul Hameed KA, Banumathi A, Ulaganathan G. Segmentation of immunohistochemical staining of $\beta$-catenin expression of oral cancer using gabor filter technique. In: Adv in Engg, Sci and Mgmt (ICAESM), 2012 Int Conf on, IEEE, 2012;429-434.

[13] Chen J, Shan S, He C, et al. WLD: A robust local image descriptor. IEEE transactions on pattern analysis and machine intelligence 2010;32(9):1705-1720.

[14] Feng Y, Ren J, Jiang J. Generic framework for content-based stereo image/video retrieval. IEEE Electronics letters. 2011;47(2):9798.

[15] Zhang Q, Izquierdo E. Histology image retrieval in optimized multi-feature spaces. IEEE J of Biomedical and Health Informatics. 2013;17(1):240-249.

[16] Manjunath BS, Ma WY. Texture features for browsing and retrieval of image data. IEEE Trans on Pattern Analysis and Machine Intelligence. 1996;18(8):837-842.

[17] Verma M, Raman B. Local tri-directional patterns: A new texture feature descriptor for image retrieval. Digital Signal Processing. 2016;51:62-72.

[18] Murala S, Maheshwari R, Balasubramanian R. Local tetra patterns: a new feature descriptor for content-based image retrieval. IEEE transactions on image processing. 2012;21(5):2874-2886. 